\title{
Estudio de la reactividad de soluciones sólidas jarosita-beudantita por sulfuración- cianuración
}

\author{
L. Sánchez ${ }^{(*)}$, A. Roca ${ }^{(*)}$, M. Cruells ${ }^{(*)}$ y J. Viñals ${ }^{(*)}$
}

\begin{abstract}
Resumen Los materiales jarosíticos pueden encontrarse en estado natural (minerales de gossan) o generarse en circuitos hidrometalúrgicos. Para recuperar la plata contenida en estos materiales, se han propuesto procesos de descomposición alcalina-cianuración a temperaturas moderadas, elevadas o a presión, según el tipo de jarosita. En este trabajo se presenta un estudio sobre el tratamiento de soluciones sólidas jarosita-beudantita con sulfuros alcalinos a temperatura ambiente, seguido de cianuración convencional para la recuperación de la plata. Las transformaciones en estado sólido se han determinado mediante difracción de rayos $\mathrm{X}$, microscopía óptica y electrónica (SEM-EDS) y microsonda electrónica. Durante la primera etapa del proceso, tiene lugar la descomposición de las fases jarosita, con formación de sulfuros de plomo y plata y un gel de hidróxido de hierro. La descomposición de potasiojarosita-beudantita tiene lugar a $p H \geq 12$ y la de beudantita-plumbojarosita, precisa de un valor más elevado $(p H=14)$. La oxidación posterior para eliminar el exceso de sulfuro, seguida de cianuración convencional, permite la recuperación de la plata contenida.
\end{abstract}

Palabras clave: Jarosita. Sulfuración. Cianuración. Gossan.

\section{Study by sulphidizing-cyanidation of jarosite-beudantite solid solutions reactivity}

\begin{abstract}
The jarositic materials occurs as ores (such as gossan) or as residues from some hydrometallurgical processes. To recover silver contained in these materials, processes of alkaline decompositioncyanidation at room or high temperatures or under pressure have been proposed. A sulphidizingcyanidation study of jarosite-beudantite solid solutions from gossan ores is presented. The solid transformations have been determined using X-ray diffraction, optical and electron microscopy (SEM-EDS) and electron microprobe. During the first step of the process the jarosite phases are decomposed with the formation of lead and silver sulphides and amorphous iron hydroxide. The decomposition of potassium jarosite-beudantite and beudantite-plumbojarosite are carried out at $p H \geq 12$ and $p H=14$, respectively. An oxidation, to remove the excess of sulphide, followed by the conventional cyanidation process leads to the recovery of the silver.
\end{abstract}

Keywords: Jarosite. Sulphidizing. Cyanidation. Gossan.

\section{INTRODUCCIÓN}

En trabajos desarrollados por este equipo de investigación ( 1 y 2 ), se ha caracterizado exhaustivamente el mineral de gossan de Río Tinto (Huelva) y estudiado el proceso de cianuración para poder explicar las recuperaciones de plata del 40-45

(*) Dpt. d’Enginyeria Química i Metal.lúrgia, Fac. de Química, Univ. de Barcelona, Martí i Franquès 1, 08028-Barcelona (España).
$\%$ obtenidas en planta industrial durante el período 1970-1996.

El mineral está constituido básicamente por goethita, hematites, cuarzo y materiales jarosíticos (soluciones sólidas de potasiojarosita-beudantita y beudantita-plumbojarosita). El oro (1-2 g/t) se encuentra en tamaño submicroscópico, aunque se han detectado algunas partículas de 0,5-1 $\mu \mathrm{m}$. La plata se presenta combinada en forma de haluros, sulfuro y sulfo-haluros de plata y mercurio, especies en las que la plata es extraible por cianuración convencional, y en soluciones sólidas de 
potasiojarosita-beudantita y beudantita-plumbojarosita, refractarias al citado proceso. La descomposición alcalina de estas especies y la subsiguiente solubilización de la plata por cianuración (2) sólo es posible en medio $\mathrm{Ca}(\mathrm{OH})_{2}$ a temperaturas elevadas $\left(80-100{ }^{\circ} \mathrm{C}\right.$, potasiojarosita-beudantita) y a presión (beudantita-plumbojarosita, $120-180{ }^{\circ} \mathrm{C}$ ) (3).

Una vía alternativa a los citados tratamientos lo constituye la sulfuración de dichas especies a $p H s$ alcalinos, oxidación del exceso de sulfuro y cianuración en medio cal a temperatura ambiente (4 y 5). El objetivo del presente trabajo es la caracterización de las transformaciones que, en estado sólido, tienen lugar durante los tratamientos de sulfuración y cianuración de las soluciones sólidas de potasiojarosita-beudantita y beudantita-plumbojarosita, contenidas en el mineral de gossan.

\section{MATERIALES Y PROCEDIMIENTO EXPERIMENTAL}

Las muestras de potasiojarosita-beudantita y beudantita-plumbojarosita se han tomado directamente del depósito de minerales de gossan de Cerro Colorado en Río Tinto (Huelva). En la tabla I (A) se incluye la composición química y la fórmula de la solución sólida de potasiojarosita-beudantita utilizada. El porcentaje de fases en la misma es el siguiente: $85,6 \%$ potasiojarosita-beudantita; 1,43 $\%$ goethita y $13,0 \%$ cuarzo. La distribución de la beudantita-plumbojarosita en el mineral ha hecho imposible la obtención directa de una muestra rica en dicha solución sólida. Por ello, el material procedente del yacimiento se ha triturado y molido a un tamaño inferior a $100 \mu \mathrm{m}$ y concentrado utilizando el licor de Thoulet $\left(\mathrm{KHgI}_{3}\right)$. La fracción densa, en la que se concentra la beudantita-plumbojarosita, representa un 9-10\% en masa de la misma, siendo el resto mayoritariamente goethita. En la tabla I (B) se incluye la composición química, obtenida por análisis mediante microsonda electrónica (EPMA), y la fórmula de los cristales de beudantita-plumbojarosita del material utilizado.

El proceso de sulfuración se ha realizado a temperatura ambiente, durante $24 \mathrm{~h}$, en un reactor agitado magnéticamente $\left(600 \mathrm{~min}^{-1}\right)$, con una solución alcalina de sulfuro potásico $(0,5 M)$ y una relación sólido/líquido de $1 \mathrm{~g}$ de solución sólida/100 ml de solución de sulfuración. Los medios de sulfuración han sido: $\mathrm{Ca}(\mathrm{OH})_{2}$ sat. y $\mathrm{NaOH}(1 M)$ para la potasiojarosita-beudantita y $\mathrm{NaOH}(1 M)$ para la beudantita-plumbojarosita. Los tratamientos realizados en probeta embutida de este material se han realizado en las mismas condiciones experimentales.

Una vez finalizada la etapa de sulfuración, los materiales se han sometido a cianuración $(2,5 \mathrm{~g}$ $\mathrm{NaCN} \mathrm{L}^{-1}$ ) durante $14 \mathrm{~h}$, con inyección de aire en el mismo sistema de reacción y una relación sólidolíquido de $1,125 \mathrm{~g} \mathrm{~L}^{-1}$ y $0,20 \mathrm{~g} \mathrm{~L}^{-1}$ para las soluciones de potasiojarosita-beudantita y beudantitaplumbojarosita, respectivamente.

El avance de las reacciones se ha seguido por difracción de rayos X (DRX), microscopía electrónica de barrido y microanálisis por energía dispersiva de rayos $\mathrm{X}$ (SEM-EDS). Los productos de sulfuración de beudantita-plumbojarosita en superficie pulida se han caracterizado por (EPMA).

\section{RESULTADOS Y DISCUSIÓN}

\subsection{Sulfuración-cianuración de potasiojarosita- beudantita.}

Los resultados obtenidos por difracción de rayos $\mathrm{X}$ del material original, del producto de sulfuración y del residuo de cianuración se presentan en la tabla II. Durante el proceso de sulfuración en medio alcalino de $\mathrm{Ca}(\mathrm{OH})_{2}$ sat, tiene lugar la descomposición de la solución sólida, con formación de sulfuro de plomo y calcita. El hierro, probablemente, se encuentra en forma de gel de hidróxidos, puesto que no se ha detectado la presencia de ningún

TABLA I.- Composición química y fórmula del: potasiojarosita beudantita (A) y beudantita-plumbojarosita (B)

TABLE I.- Chemical composition and formula of: potassium jarosite beudantite (A) and beudantiteplumbojarosite $(B)$

\begin{tabular}{|ccccccccc|}
\hline Material A & Elemento & $\mathrm{Fe}$ & $\mathrm{S}$ & $\mathrm{As}$ & $\mathrm{Pb}$ & $\mathrm{K}$ & $\mathrm{Na}$ & $\mathrm{Ag}$ \\
\cline { 2 - 8 } & $(\%)$ & 26.5 & 7.30 & 2.78 & 9.43 & 3.48 & 0.21 & 143 \\
& Fórmula & $\left(\mathrm{Pb}_{0.30} \mathrm{~K}_{0.59} \mathrm{Na}_{0.06}\right)$ & $\mathrm{Fe}_{3}\left(\mathrm{SO}_{4}\right)_{1.76}\left(\mathrm{AsO}_{4}\right)_{0.24}(\mathrm{OH})_{6}$ & & \\
\hline Material B & Elemento & $\mathrm{Fe}$ & $\mathrm{As}$ & $\mathrm{S}$ & & $\mathrm{Pb}$ & & $\mathrm{Ag}$ \\
& & $(\%)$ & 24.7 & 8.50 & 5.79 & 27.2 & 850 \\
& \multirow{2}{*}{ Fórmula } & $\left(\mathrm{Pb}_{0.84} \mathrm{Ag}_{0.085}\right) \mathrm{Fe}_{3}\left(\mathrm{SO}_{4}\right)_{1.23}\left(\mathrm{AsO}_{4}\right)_{0.77}(\mathrm{OH})_{6}$ & & \\
& & &
\end{tabular}

* ppm 
TABLA II.- Potasiojarosita-beudantita: resultados de difracción de rayos-X

TABLE II.- Potassium jarosite-beudantite: results of $X$-ray diffraction

\begin{tabular}{|l|c|c|c|}
\hline Especies & $\begin{array}{c}\text { Material } \\
\text { original }\end{array}$ & $\begin{array}{c}\text { Material } \\
\text { sulfurado }\end{array}$ & $\begin{array}{c}\text { Material } \\
\text { cianurado }\end{array}$ \\
\hline Mayoritarias & $\begin{array}{c}\text { potasiojarosita- } \\
\text { beudantita }\end{array}$ & - & calcita \\
\hline Minoritarias & $\begin{array}{c}\text { goethita } \\
\text { cuarzo } \\
\text { hematies }\end{array}$ & $\begin{array}{c}\text { PbS, goethita } \\
\text { cuarzo, calcita } \\
\text { hematies }\end{array}$ & $\begin{array}{c}\text { goethita } \\
\text { cuarzo } \\
\text { hematies }\end{array}$ \\
\hline
\end{tabular}

producto de este elemento en fase cristalina. Durante la cianuración con inyección de aire, el sulfuro de plomo se oxida y se forma una mayor cantidad de calcita. En los residuos de cianuración, no se detectan, por dicha técnica, fases cristalinas de hierro o de plomo.

Mediante la técnica de SEM se ha observado (Fig. 1), la morfología de las partículas de potasiojarosita-beudantita (cristales romboédricos) y su tamaño $(1-5 \mu \mathrm{m})$.

Comparando los espectros EDS correspondientes a la solución sólida (Fig. 2) y a los productos de descomposición (Fig. 3), se observa una importante disminución de la intensidad de las líneas $K_{\alpha}$ del azufre, del potasio y del arsénico, mientras que las líneas $K_{\alpha}$ y $K_{\beta}$ del hierro no sufren modificación.

La reacción de sulfuración en las condiciones experimentales descritas puede escribirse como:

$$
\begin{gathered}
\left(\mathrm{Pb}_{0,30} \mathrm{~K}_{0,59} \mathrm{Na}_{0,06}\right) \mathrm{Fe}_{3}\left(\mathrm{SO}_{4}\right)_{1,76}\left(\mathrm{AsO}_{4}\right)_{0,24} \\
(\mathrm{OH})_{6(\mathrm{~s})}+0,30 \mathrm{~S}^{2-}{ }_{(\mathrm{aq})}+3 \mathrm{OH}^{-}{ }_{(\mathrm{aq})} \longrightarrow
\end{gathered}
$$

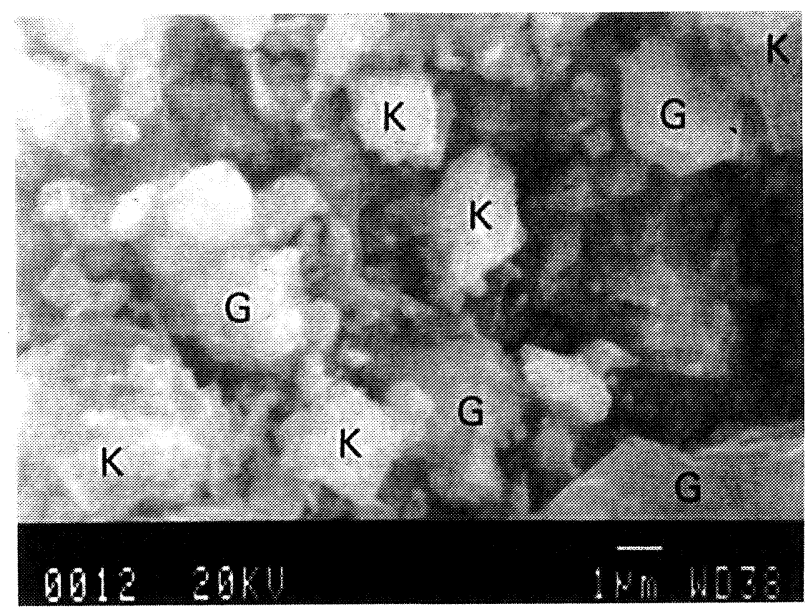

FIG. 1.- Imagen SEM-SE de potasiojarositabeudantita $(\mathrm{K})$ en goethita $(\mathrm{G})$.

FIG. 1.- SEM-SE image of potassiumjarositebeudantite, $(K)$ in goethite $(G)$.

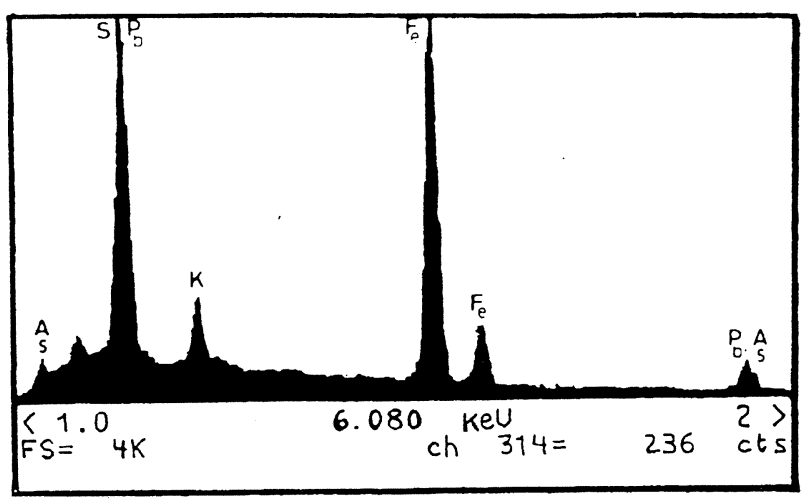

FIG. 2.- Espectro EDS de potasiojarosita-beudantita.

FIG. 2.- EDS spectrum of potassiumjarositebeudantite.

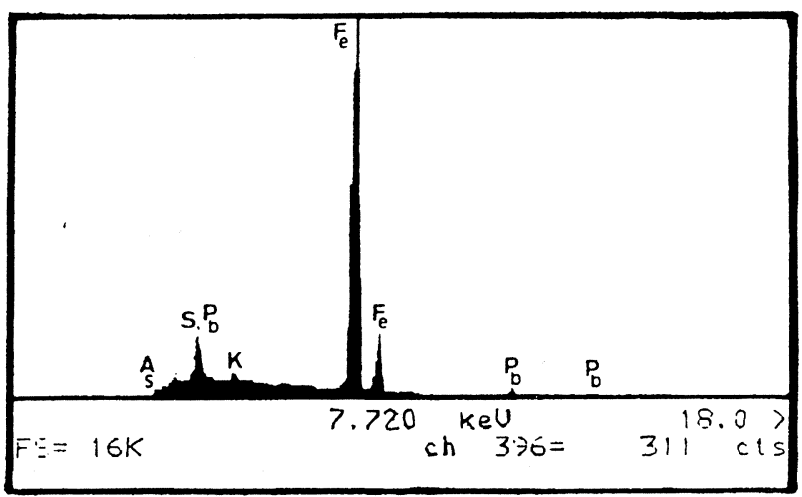

FIG. 3.- Espectro EDS del producto de sulfuración.

FIG. 3.-EDS spectrum of sulphidized product.

$$
\begin{gathered}
0,30 \mathrm{PbS}_{(\mathrm{s})}+0,59 \mathrm{~K}^{+}{ }_{(\mathrm{aq})}+0,06 \mathrm{Na}^{+}{ }_{(\mathrm{aq})}+3 \mathrm{Fe}(\mathrm{OH})_{3(\mathrm{~s})^{+}} \\
+1,76 \mathrm{SO}_{4}{ }^{2-}{ }_{(\mathrm{aq})}+0,24 \mathrm{AsO}_{4}{ }^{3-}{ }_{(\mathrm{aq})}
\end{gathered}
$$

En presencia de un exceso de sulfuro las especies $\mathrm{Fe}(\mathrm{III})$ y $\mathrm{As}(\mathrm{V})$ pueden sufrir reacciones secundarias y dar lugar a Fe(II) y tiosales de arsénico.

Los resultados obtenidos por difracción de rayos $\mathrm{X}$ del material original, del producto de sulfuración y del residuo de cianuración se presentan en la tabla III.

TABLA III.- Beudantita-plumbojarosita: resultados de difracción de rayos-X

TABLE III.- Beudantite-plumbojarosite: results of $X$-ray diffraction

\begin{tabular}{|l|c|c|c|}
\hline Especies & $\begin{array}{c}\text { Material } \\
\text { original }\end{array}$ & $\begin{array}{c}\text { Material } \\
\text { sulfurado }\end{array}$ & $\begin{array}{c}\text { Material } \\
\text { cianurado }\end{array}$ \\
\hline Mayoritarias & $\begin{array}{c}\text { goethita, cuarzo } \\
\text { beudantita-PbJar }\end{array}$ & $\begin{array}{c}\text { goethita, } \\
\text { cuarzo } \\
\text { PbS }\end{array}$ & $\begin{array}{c}\text { goethita } \\
\text { cuarzo }\end{array}$ \\
\hline Minoritarias & barita & barita & barita \\
\hline
\end{tabular}


Durante el proceso de sulfuración en medio $\mathrm{NaOH}$ tiene lugar la descomposición de la solución sólida de beudantita-plumbojarosita con formación de sulfuro de plomo. No se detectan especies cristalinas de hierro, lo que induce a pensar en una probable formación de un gel de hidróxido de hierro. En la etapa de cianuración, el sulfuro de plomo se oxida, no detectándose en el residuo de esta etapa especies cristalinas de hierro o de plomo.

Por microscopía óptica de reflexión sobre superficie pulida, se pone de manifiesto (Fig. 4) el avance de la reacción de sulfuración en medio $\mathrm{NaOH}$ $(1 M)$. En la misma, se observa la morfología (cristales romboédricos) de las partículas de beudantitaplumbojarosita antes de iniciarse la sulfuración (Fig. 4 a) y cómo la forma zonal de los cristales originales persiste a lo largo de la reacción. En la figura 5 se presenta una imagen SEM-SE del producto de sulfuración de la solución sólida $(t=24 \mathrm{~h})$.

Los espectros EDS de las muestras de beudantita-plumbojarosita (Fig. 6) y de su producto de sulfuración, en medio $\mathrm{NaOH}(1 M)$ durante $24 \mathrm{~h}$ (Fig. 7 ), ponen de manifiesto una disminución muy
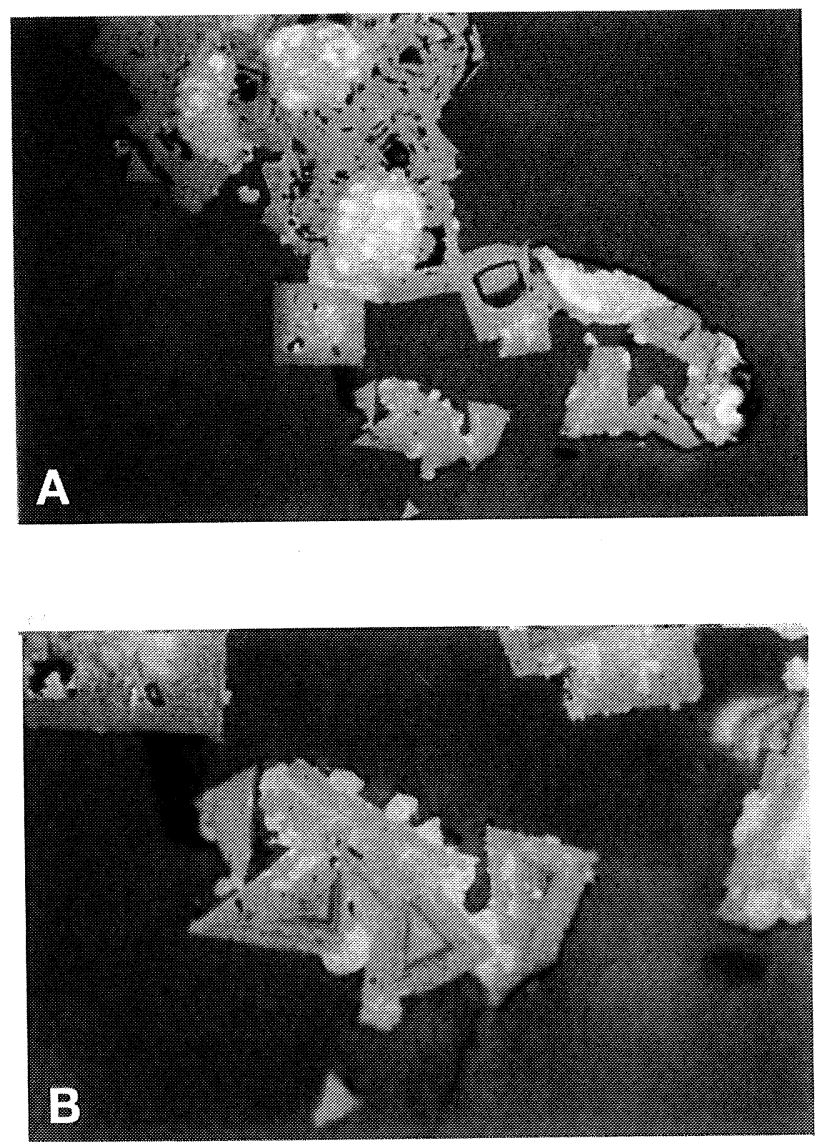

importantes de las líneas $K_{\alpha}$ del azufre y del arsénico y de la línea $L_{\alpha}$ del arsénico; mientras que las líneas $K_{\alpha}$ y $K_{\beta}$ del hierro no experimentan variación.

En la tabla IV se presenta la media de 20 análisis realizados por EPMA sobre partículas de beudantita-plumbojarosita y de su producto de sulfuración.

La reacción de sulfuración de beudantita-plumbojarosita en las condiciones experimentales descritas puede expresarse como:

$$
\begin{aligned}
\mathrm{Pb}_{0,84} \mathrm{Ag}_{0,085} \mathrm{Fe}_{3}\left(\mathrm{SO}_{4}\right)_{1,23}\left(\mathrm{AsO}_{4}\right)_{0,77}(\mathrm{OH})_{6(\mathrm{~s})}+ \\
\quad+0,88 \mathrm{~S}^{2-}{ }_{(\mathrm{aq})}+3 \mathrm{OH}^{-}{ }_{(\mathrm{aq})} \longrightarrow \\
\\
0,043 \mathrm{Ag}_{2} \mathrm{~S}_{(\mathrm{s})}+0,84 \mathrm{PbS}_{(\mathrm{s})}+3 \mathrm{Fe}(\mathrm{OH})_{3(\mathrm{~s})} \\
+1,23 \mathrm{SO}_{4}{ }^{2-}{ }_{(\mathrm{aq})}+0,77 \mathrm{AsO}_{4}{ }^{3-}{ }_{(\mathrm{aq})}
\end{aligned}
$$

A su vez, las especies Fe(III) y As(V), en presencia de un exceso de sulfuros, pueden sufrir reacciones secundarias y dar lugar a Fe(II) y tiosales de arsénico.
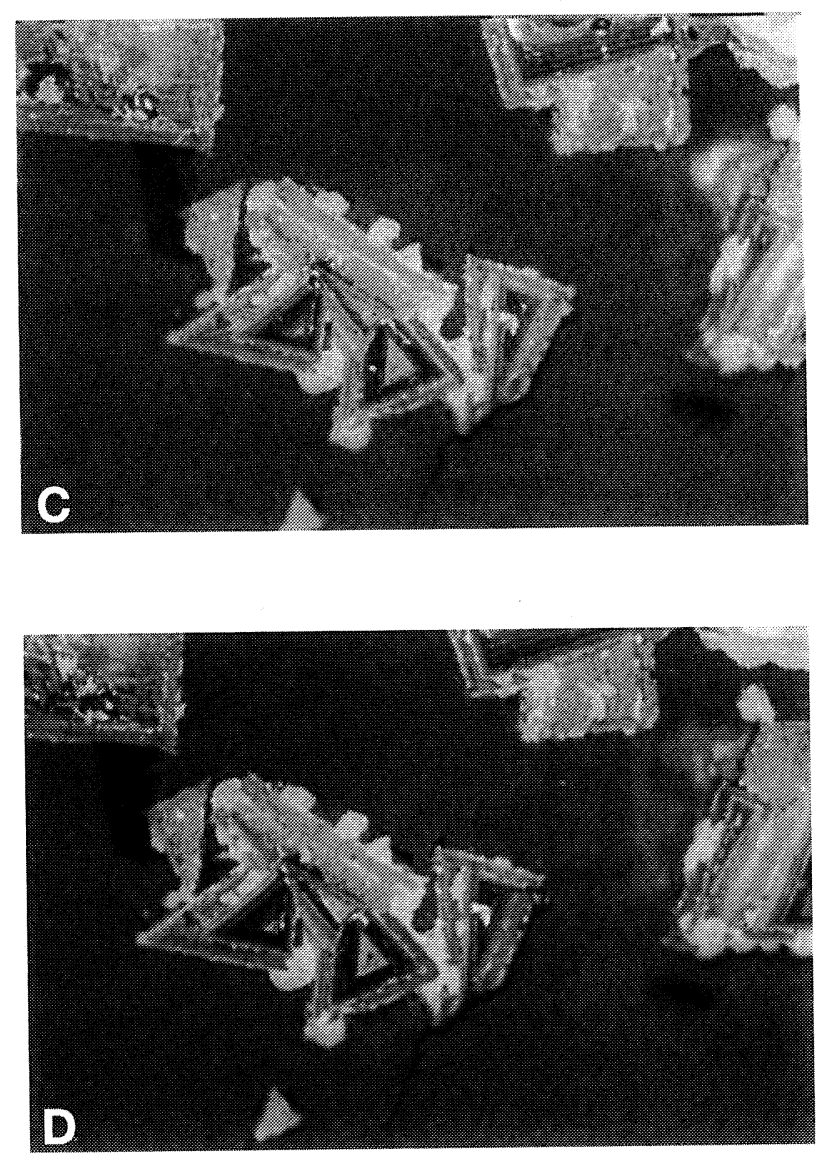

Fig. 4. - Sulfuración de beudantita-plumbojarosita a $25^{\circ} \mathrm{C}$ en $\mathrm{NaOH} 1 M . A$ ) muestra original, $\left.\times 85 . B\right) 2 \mathrm{~h}$. C) 12 h. D) 24 h. Imágenes por M.O. de reflexión sobre superficie pulida, $\times 215$.

FIG. 4.- Sulphidizing of beudantite-plumbojarosite at $25^{\circ} \mathrm{C}$ in $\mathrm{NaOH} 1 \mathrm{M}$. A) original sample, $\left.\times 85 . \mathrm{B}\right) 2 \mathrm{~h}$. C) 12 h. D) 24 h. Images obtained by reflected O.M. over polished surphace, $\times 215$. 


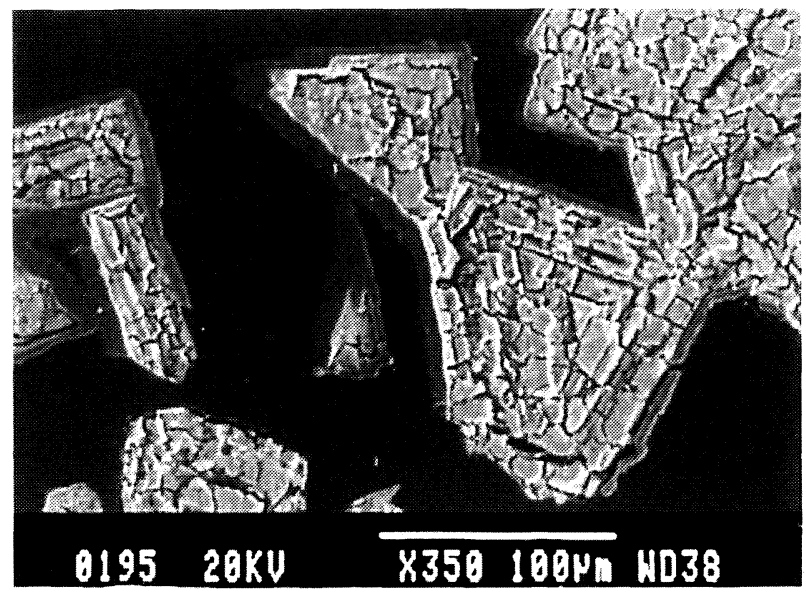

Fig. 5.- Muestra sulfurada de beudantitaplumbojarosita (SEM).

FIG. 5.- Sulphidized sample of beudantiteplumbojarosite (SEM).

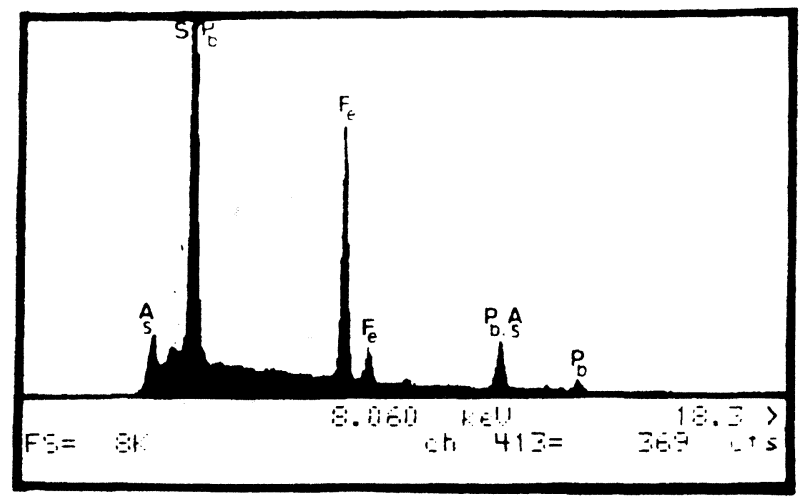

FIG. 6.- Espectro EDS de la beudantita-plumbojarosita.

FIG. 6.- EDS spectrum of beudantiteplumbojarosite.

\section{CONCLUSIONES}

Las transformaciones que tienen lugar en el sólido durante la sulfuración y subsiguiente cianuración de soluciones sólidas de potasiojarosita-beudantita y beudantita-plumbojarosita son:

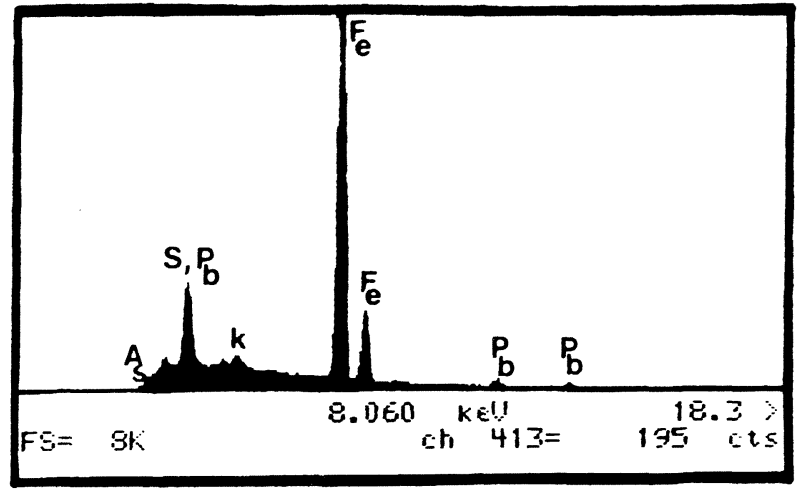

FIG. 7.- Espectro EDS del producto de sulfuración.

FIG. 7.-EDS spectrum of sulphidized product.

- La sulfuración de la potasiojarosita-beudantita, en medio saturado de cal y temperatura ambiente, conduce a la formación de sulfuros de plomo y de plata y a un gel de hidróxido de hierro. Los iones sulfato, arseniato y potasio pasan a la solución. En la cianuración subsiguiente, tiene lugar la oxidación del sulfuro de plomo y la complejación de la plata.

- La sulfuración de la beudantita-plumbojarosita en medio $\mathrm{NaOH}$ y a temperatura ambiente, así como la cianuración subsiguiente, conduce a las mismas transformaciones que en el caso anterior. Sin embargo, se requiere un $p H=14$ y largos tiempos de residencia $(24 \mathrm{~h})$.

\section{REFERENCIAS}

(1) Viñals, J., Roca, A., Cruells, M. y Nuñez, C. Can. Met. Q., 34 (2), 1995: 115-122.

(2) Roca, A., Viñals, J., Arranz, M. y Calero, J. Can. Met. $Q$. Pendiente de publicación, 1997.

(3) Arranz, M. Tesis Doctoral. Presentación 1998.

(4) NuÑEz, C., RocA, A. y EsPiell, F. US Patent n ${ }^{\circ} 4.654 .079$. 1987.

(5) Sanchez, L., Cruells, M. y Roca, A. Hydrometallurgy, 42, 1996: 35-49.

TABLA IV.- Beudantita-plumbojarosita: resultados de EPMA

TABLE IV.-Beudantite-plumbojarosite: results of EPMA

\begin{tabular}{|lccccccc|}
\hline Especie / Elementos $(\%)$ & $\mathrm{O}$ & $\mathrm{Pb}$ & $\mathrm{S}$ & $\mathrm{Fe}$ & $\mathrm{Cu}$ & $\mathrm{As}$ & $\mathrm{K}$ \\
\hline Beudantita-Pbjarosita & 32,6 & 29,1 & 4,38 & 21,7 & 1,01 & 9,76 & - \\
Producto de sulfuración & 21,1 & 38,6 & 6,94 & 28,8 & 1,19 & 0,17 & 1,12 \\
\hline
\end{tabular}

